\title{
Großräumige grenzüberschreitende Verflechtungsräume
}

\author{
Ein „Modellvorhaben der Raumordnung“ (MORO) des Bundes
}

\author{
Andrea Hartz · Gerd-Rainer Damm · Stefan Köhler
}

Eingegangen: 1. Februar 2010 / Angenommen: 19. Oktober 2010 / Online publiziert: 19. November 2010

(C) Springer-Verlag 2010

Zusammenfassung Im Rahmen eines Modellvorhabens des Bundes wird die Rolle wirtschaftsstarker Grenzregionen im Zusammenspiel mit europäischen Metropol- und Stadtregionen untersucht. Gemeinsam mit Partnern aus der Euregio Maas-Rhein, der (europäischen) Großregion (SaarLorLux + ), der Trinationalen Metropolregion Oberrhein sowie der Bodenseeregion verfolgt die Bundesraumordnung das Ziel, die besondere Bedeutung grenzüberschreitender Verflechtungsräume für Wachstum und Innovation sowie für das europäische Ziel der territorialen Kohäsion in der raumordnungspolitischen Diskussion in Deutschland und Europa zu etablieren. Ein wesentlicher Aspekt stellt die Erprobung des strategischen Ansatzes der ,großräumigen Verantwortungsgemeinschaften“ im grenzüberschreitenden Kontext dar. Zudem richtet sich das Interesse der Partnerschaft auf die Regionen selbst. Das Projekt soll eine kohärente Regionalentwicklung sowie effektive Kooperationsstrukturen über nationalstaatliche Grenzen hinweg stärken. Schließlich verfolgt das Projekt die Gründung eines Initiativkreises als offenes Bündnis der grenzüberschreitenden Verflechtungsräume, um die Anliegen der Grenzregionen auf nationaler und europäischer Ebene wirksam zu vertre-

\footnotetext{
A. Hartz $(\bowtie)$

Planungsgruppe agl, Großherzog-Friedrich-Straße 47, 66111 Saarbrücken, Deutschland

E-Mail: andreahartz@agl-online.de

G.-R. Damm

Ministerium für Umwelt, Energie und Verkehr des Saarlandes, Keplerstraße 18, 66117 Saarbrücken, Deutschland

E-Mail: g.damm@umwelt.saarland.de

Dr. S. Köhler

Stadt Friedrichshafen, Adenauerplatz 1, 88045 Friedrichshafen, Deutschland

E-Mail: s.koehler@friedrichshafen.de
}

ten. Der Beitrag liefert einen Einblick in die Genese, Zielsetzung und Vorgehensweise dieses Modellvorhabens vor dem Hintergrund aktueller Diskussionen um eine zukunftsfähige Raumentwicklung in Deutschland und Europa.

Schlüsselwörter Grenzüberschreitende

Verflechtungsräume · Großräumige

Verantwortungsgemeinschaften · Modellvorhaben der Raumordnung · Territoriale Kohäsion · Europäische Integration

\section{Large-Scale Cross-Border Metropolitan Regions}

\section{A Demonstration Project of Spatial Planning (MORO)}

\begin{abstract}
A federal pilot project will explore the role played by economically strong border regions in interaction with European metropolitan and urban regions. Jointly with partners from the Maas-Rhine Euroregion, (SaarLorLux+) Euroregion, Trinational Upper Rhine metropolitan area and Lake Constance region, the regional policy of the federal government is aimed at establishing the particular importance of cross-border metropolitan areas for growth and innovation, as well as for the European target of territorial cohesion, within regional policy discussions in Germany and Europe. Testing the strategic approach of "supra-regional partnerships" in a cross-border context, is one significant aspect. The interests of the partners involved in the project are furthermore focussed on the regions themselves. This means, the project is dedicated to promote a coherent regional development, as well as effective cooperation structures across national borders. The project finally pursues the creation of an initiative in the form of an open alliance of cross-border metropolitan regions, in order to more effectively represent the interests
\end{abstract}


of border regions on a national and European level. The article explores the genesis, objectives and approach of this pilot project against the background provided by current debates on sustainable spatial development in Germany and Europe.

Keywords Cross-border metropolitan regions .

Supra-regional partnerships $\cdot$ Cross-border governance $\cdot$

Territorial cohesion $\cdot$ European integration

\section{Einführung: Grenzregionen und nationale Raumentwicklungspolitik}

Vom jeweiligen Nationalstaat aus gesehen liegen Grenzregionen ,am Rand'. Die Ausrichtung von politischer Macht und wirtschaftlichem Wachstum konzentrierte sich in den letzten Jahrhunderten stärker auf die nationalstaatlichen Zentren. Grenzräume wurden als Peripherie marginalisiert und verfolgten in ihrer Entwicklung gleichfalls eine nationalstaatliche Orientierung. Dies schwächt den Integrationsprozess in den Grenzregionen und damit deren Wettbewerbsfähigkeit bis heute.

Auch wenn sich seit dem Ende des Zweiten Weltkrieges durch die Entwicklung der EU, das Schengener Abkommen und insbesondere durch den Fall des „Eisernen Vorhangs“ die Bedeutung nationalstaatlicher Grenzen im Zuge von Globalisierung und europäischer Integration grundsätzlich gewandelt hat, werden Grenzräume aufgrund ihrer dezentralen Lage selbst dann in den nationalen Raumentwicklungspolitiken nicht ausreichend wahrgenommen, wenn sich grenzüberschreitende Wachstumsregionen und intensive Verflechtungen ausbilden konnten. Dies zeigt ein Blick auf die Karte „Wachstum und Innovation“ der „Neuen Leitbilder und Handlungsstrategien" von Bund und Ländern, die grenzüberschreitende Verflechtungen nicht abbildet (BMVBS/BBR 2006: 40), gleichwohl die besondere Rolle grenzüberschreitender Verflechtungsräume bereits (an)erkennt und in die Weiterentwicklung der Leitbilder einbeziehen will: „Das raumordnerische Metropolregionenkonzept wird $u$. a. vor dem Hintergrund der zunehmenden Bedeutung grenzüberschreitender europäischer metropolitaner Verflechtungsräume, wie z. B. am Oberrhein und im deutsch-belgisch-niederländischen Grenzraum, weiterentwickelt" (BMVBS/BBR 2006: 44).

Im Zuge der aktuellen Diskussionen um die Lissabonund Göteborg-Strategien sowie das europäische Ziel der territorialen Kohäsion, um Metropolregionen und großräumige Verantwortungsgemeinschaften erscheint es deshalb notwendig, die spezifischen Entwicklungspotenziale der grenzüberschreitenden Verflechtungsräume näher zu betrachten und in den Fokus nationaler und europäischer Politiken zu rücken. Dieser Aufgabe widmet sich derzeit ein Modellvorhaben der Raumordnung (MORO) des Bundes.
Genese, Zielsetzung und Vorgehensweise dieses Modellvorhabens werden nachfolgend - vor dem Hintergrund aktueller Diskussionen um Raumentwicklung in Deutschland und Europa - dargelegt. Es wird ein Überblick zu bisherigen Ergebnissen, beispielsweise hinsichtlich einer Definition grenzüberschreitender Verflechtungsräume und den besonderen Rahmenbedingungen grenzüberschreitender Regionalentwicklung, gegeben.

\section{Bedeutung und Rolle der Grenzregionen}

Über $30 \%$ der Bevölkerung der erweiterten EU leben in Grenzräumen, die mehr als $40 \%$ des EU-Territoriums einnehmen (AGEG ${ }^{1}$ 2004). Bereits diese Zahlen machen die Bedeutung von Grenzregionen bewusst. Grenzregion ${ }^{2}$ meint in diesem Kontext einen identifizierbaren Handlungsraum (Region), der sich durch eine oder mehrere nationalstaatliche Grenzen und damit durch nationalstaatliche Teilräume auszeichnet und gleichzeitig durch grenzüberschreitende institutionelle Arrangements auf subnationaler Ebene repräsentiert wird (vgl. hierzu Schmitt-Egner 2005). Grenzregionen sind „Subsysteme, die sich aus der horizontalen Vernetzung (und punktuellen Integration) von funktionalen Teilbereichen der jeweils in Frage stehenden nationalen Referenz-Systeme konstituieren“ (Beck 2010: 25).

Nationalstaatliche Grenzen wirken hierbei in besonderer Weise als Barrieren und als Schnittstellen, wobei sich die Bedeutung der äußeren Grenzen eines Staates erst mit der Entstehung moderner Nationalstaaten herausgebildet und in der heute gelebten Form verfestigt hat: Seit der Ausbildung moderner Nationalstaaten ,diente die Grenze sowohl im funktionellen als auch im symbolischen Sinne als ein konstitutives Mittel zur deutlichen Einfassung des eigenen Staatsgebietes und zur klar definierten Abgrenzung von anderen Staatseinheiten“ (Krocová 2007: 182). In den letzten Dekaden beförderten Globalisierung und europäischer Integrationsprozess „,die Diskussion darüber, ob Grenzen überholt sind oder ob sie neu definiert werden müssen - als komplexe und vielschichtige innere Grenzen einer integrierten Welt" (Duhamelle/Kossert/Struck 2007: 9). Dabei wächst das Interesse an den Akteuren und Grenzgesellschaften, die mit ihren „Logiken, Wahrnehmungen und Interaktionen“ einen differenzierten Umgang mit den unterschiedlichen Aus-

\footnotetext{
${ }^{1}$ Die Arbeitsgemeinschaft Europäischer Grenzregionen (AGEG) wurde 1971 gegründet; sie vertritt die Interessen der Grenz- und grenzüberschreitenden Regionen und betreibt eine aktive Informations- und Netzwerkpolitik. Mittlerweile zählt die Vereinigung 90 Mitglieder in ganz Europa; vgl. www.aebr.net (letzter Zugriff am 03.06.2009).

${ }^{2}$ Im Gegensatz zu Grenzregion als identifizierbarer Handlungsraum betont der Begriff „Grenzraum“ aus Sicht der Autoren lediglich die Grenzlage eines Raumes, ohne dass damit eine spezifische Form von Verfasstheit gemeint ist.
} 
prägungen und Wirksamkeiten nationalstaatlicher Grenzen pflegen, enorme Transferleistungen zwischen nationalstaatlichen Systemen erbringen und im Kontext lebensweltlicher und alltäglicher Praktiken die Grenze gleichsam beständig reproduzieren (Duhamelle/Kossert/Struck 2007: 10).

In der europäischen Politik entfachten erst die deutsche Wiedervereinigung, der Zusammenbruch der ehemaligen Sowjetunion und seine Implikationen für das nationalstaatliche Gefüge sowie die EU-Osterweiterung mitsamt all ihren Folgen ein verstärktes Interesse an Grenzregionen. Bis dahin blieben Grenzregionen eher ein Randthema.

Bis 1987 standen ,nur in sehr geringem Umfang Gemeinschaftsmittel aus dem Bereich der Regionalpolitik für Aktionen der grenzüberschreitenden Zusammenarbeit zur Verfügung“ (Manthey 1992: 31). Zwischen 1980 und 1987 wurden weniger als eine Million ECU hierfür aufgewendet (Manthey 1992: 31). Dies änderte sich 1990 grundlegend mit der Einführung der europäischen Gemeinschaftsinitiative INTERREG als eigene Förderkulisse für grenzüberschreitende (A-Programm), transnationale (B-Programm) und interregionale (C-Programm) Zusammenarbeit, die für einen enormen Schub an gemeinsamen grenzüberschreitenden Programmen, Projekten und Initiativen sorgte. Die INTERREG-Programme förderten dabei nicht nur das Denken und Agieren über nationale Grenzen hinweg und in gröBeren transnationalen Kooperationsräumen, sondern auch die Erkenntnis der strategischen Notwendigkeit, „über eine räumlich kohärente Koordination der Gemeinschaftspolitiken die europäische Integration zu fördern“ (Battis/Kersten 2008: 7).

Neben projektbezogenen Aktivitäten führte die grenzüberschreitende Zusammenarbeit zu strategisch orientierten und verstetigten Organisationsstrukturen. Hierbei lassen sich unterschiedliche und häufig sehr komplexe, sich überlagernde Institutionalisierungsprozesse beobachten: Diese reichen von der Gründung informeller Arbeitsgemeinschaften und Vereinsstrukturen auf lokaler und regionaler Ebene bis zu „Euroregionen“ und Eurodistrikten. ${ }^{3}$

Mit dem Karlsruher Übereinkommen von 1996, das unter anderem die Gründung grenzüberschreitender örtlicher

\footnotetext{
${ }^{3}$ Unter Euroregionen (oder Euregios) versteht die Arbeitsgemeinschaft Europäischer Grenzregionen (AGEG 2000: A1-8, B2-22) einen nach dem Privat- oder öffentlichen Recht organisierten Zusammenschluss regionaler und lokaler Körperschaften beiderseits einer Staatsgrenze mit eigenen administrativen, personellen und finanziellen Ressourcen, teilweise mit parlamentarischer Versammlung. Eurodistrikte umfassen ausschließlich Verflechtungsräume - in Abgrenzung zu den Euroregionen oder Euregios, die auch periphere und eher ländlich strukturierte Regionen einbeziehen. Eurodistrikte sehen sich in der konkreten Umsetzung durchaus mit erheblichen Schwierigkeiten konfrontiert, beispielweise hinsichtlich der räumlichen Abgrenzung - inwieweit diese als zumeist politische Setzung auch den tatsächlichen Verflechtungen entspricht - oder hinsichtlich der Frage, welche Kompetenzen an den Eurodistrikt übertragen werden können (Levin 2006).
}

Zweckverbände entlang der Grenzen zwischen Deutschland, Frankreich, Luxemburg und der Schweiz regelt, sowie der deutsch-französischen Erklärung zum 40. Jahrestag des Élysée-Vertrages von 2003, die zu einer Intensivierung der grenzüberschreitenden Kooperation im Rahmen von Eurodistrikten aufruft, erhielt die Institutionalisierung grenzüberschreitender Strukturen neuen Schwung. Die Erklärung bezieht sich zunächst explizit auf die Schaffung eines Eurodistrikts Straßburg-Kehl, ruft aber gleichzeitig zur Gründung weiterer Eurodistrikte auf.

Seit den 1990er Jahren gewinnen somit sowohl die Grenzregionen als auch die Raumakteure an strategischer Relevanz für die europäische Integration. In der vierten INTERREG-Förderperiode von 2007 bis 2013 wurde aus der Gemeinschaftsinitiative ein „Mainstream-Programm“ der europäischen Strukturpolitik: Das neue Ziel 3 „Europäische territoriale Zusammenarbeit" (INTERREG IV) macht einmal mehr deutlich, dass den räumlichen Bedingungen der wirtschaftlichen und sozialen Entwicklung mehr Aufmerksamkeit gewidmet wird (Ahlke/Görmar/Hartz 2007: 451). Nach Battis und Kersten (2008: 7) zeichnet sich in den Politiken der EU seit den 1990er Jahren ein spatial turn ab, der in einem raumstrategischen Integrationsdenken und der Betonung des territorialen Zusammenhalts als wesentliches Ziel seinen Niederschlag findet. Das dreidimensionale Konzept des territorialen Zusammenhalts bezieht sich auf den territorialen Ausgleich als Ausgleich räumlicher Ungleichgewichte, die territoriale Integration als horizontale und vertikale Koordination raumrelevanter Politiken sowie die territoriale Governance zur Vernetzung europäischer, nationaler und subnationaler Raumakteure. Alle Dimensionen besitzen eine hohe Relevanz für die grenzüberschreitenden Verflechtungsräume und sind Anknüpfungspunkt für deren weitere Entwicklung. Battis und Kersten (2008: 14) verweisen zudem völlig zu Recht auf den Zweiten Kohäsionsbericht (European Commission 2001), der festhält, dass die grenzüberschreitende, transnationale und interregionale Zusammenarbeit eine „Schlüsselpriorität“ für die europäische Integration und für eine Verringerung der ökonomischen und sozialen Fragmentierung, die durch nationalstaatliche Grenzen verursacht wird, darstellt.

Gleichzeitig öffnet sich hier das Spannungsfeld zwischen „entgrenzten sozialen und wirtschaftlichen Beziehungen einerseits und politischen Verantwortungsräumen andererseits" (Battis/Kersten 2008: 7). Die funktionale Schwächung nationalstaatlicher Grenzen wird zum Symbol dieses Spannungsfeldes; grenzübergreifende Kooperationen und Regionalisierungsprozesse, das heißt die Ausbildung und das Funktionieren neuer Handlungsräume jenseits nationalstaatlicher Einheit und Regelungsmechanismen, werden damit zum Experimentierfeld von European Territorial Governance. 
Die grenzüberschreitende Zusammenarbeit besitzt deshalb eine Schlüsselrolle bei der Anwendung des Europäischen Raumentwicklungskonzeptes (Europäische Kommission 1999: 180). Das Europäische Raumentwicklungskonzept fordert eine Intensivierung der Bemühungen um integrierte Raumentwicklungsstrategien über nationalstaatliche Grenzen hinweg: „Integrierte Raumentwicklungspolitik im EU-Maßstab muß die politischen Optionen zur Entwicklung bestimmter Gebiete so miteinander kombinieren, daß nationale Grenzen und andere administrative Hürden keine Entwicklungsengpässe mehr darstellen“ (Europäische Kommission 1999: 162). Auf dem informellen Ministertreffen zur Stadtentwicklung und zum territorialen Zusammenhalt am 24. und 25. Mai 2007 in Leipzig wurde die „Territoriale Agenda der EU“ verabschiedet. Sie zeigt Handlungsmöglichkeiten und -ansätze auf, um die Ziele des Europäischen Raumentwicklungskonzeptes mit den Lissabon- und Göteborg-Strategien zu synchronisieren (Ahlke/Görmar/Hartz 2007: 450). Die grenzüberschreitende Kooperation spielt in allen angesprochenen Politikfeldern eine wesentliche Rolle. Beide Dokumente bilden, zusammen mit dem Grünbuch zum territorialen Zusammenhalt (Kommission der Europäischen Gemeinschaft 2008), das Fundament für die Ausrichtung der Gemeinschaftspolitiken an einer kohärenten Entwicklung des EU-Territoriums.

Grenzregionen gelten in diesem Kontext als „Schnittstellen“ bzw. „Scharniere“ zwischen den nationalstaatlichen Sphären, gleichzeitig als „Labore“ der konkreten und erfolgreichen Umsetzung der territorialen Integration (vgl. hierzu Beck 2010). Im Rahmen der europäischen Integration kommt ihnen eine besondere Rolle zu - als Experimentierfeld für die europäische Raumentwicklung, für das Überwinden nationalstaatlicher Barrieren und für die Erfahrung eines „kleinen Europas“ im Alltag der Bürgerinnen und Bürger.

\section{Grenzregionen im Kontext nationalstaatlicher Raumordnung}

Es stellt sich nun die Frage, inwieweit die nationalen Raumordnungspolitiken der Bedeutung der Grenzregionen für das Gelingen der europäischen Integration ausreichend Rechnung tragen. Dazu werden die neuen Leitbilder der Raumordnung in Deutschland, aber auch die Konzepte einiger Nachbarstaaten kritisch beleuchtet, nicht zuletzt, um eine vergleichende Einschätzung aktueller Politiken zu ermöglichen.

Die nachfolgenden Ausführungen zeigen den Handlungsbedarf auf, der sich im Spannungsfeld zwischen europäischen Politiken und nationalen Interessen ergibt, wenn es darum geht, Grenzräume angemessen in den nationalen Kontexten zu berücksichtigen und vom Rand in den Fokus zu rücken. In Bezug auf die neuen Leitbilder des Bundes und der Länder in Deutschland lässt sich dies sehr gut an der Debatte über Metropolregionen nachvollziehen. Inwieweit die Grenzregionen in dieser Debatte eine Rolle spielen, soll hier näher betrachtet werden.

\subsection{Die neuen Leitbilder der Raumordnung in Deutschland}

Bund und Länder setzen mit den drei neuen Leitbildern „Wachstum und Innovation“, „Daseinsvorsorge sichern“ sowie „Ressourcen bewahren, Kulturlandschaften gestalten“ Schwerpunkte für eine nachhaltige Raumentwicklungspolitik in Deutschland (BMVBS/BBR 2006: 8). Zentrale Bausteine des Leitbildes „Wachstum und Innovation“ sind die Konzepte der „Europäischen Metropolregionen“ und der „Großräumigen Verantwortungsgemeinschaften“.

Im Rahmen der Umsetzung der Lissabon-Strategie für mehr Wachstum und Innovation in Europa und als eine Antwort auf die neuen Herausforderungen der Globalisierung gewinnen die Metropolregionen als wirtschaftsstarke Räume zunehmend an Bedeutung. Blotevogel (2007: 9) sieht weitere Gründe für den Bedeutungsgewinn in der Schwächung der territorialen Logik der Nationalstaaten und -ökonomien sowie in einer Stärkung der Netz-Knoten-Logik durch neue Transport- und Kommunikationstechniken und in der Globalisierung der Märkte. Knotenpunkte und Impulsgeber dieser weltweiten Verflechtungen sind die metropolitanen Ökonomien mit einem spezifischen Mix aus Wachstumsbranchen im Dienstleistungssektor. Blotevogel (2007: 10) weist hierbei auf ein Paradoxon hin: Der Entgrenzung der Märkte und Flexibilisierung von Realkapital und (kodifiziertem) Wissen steht eine hochgradige Lokalisierung von implizitem Wissen (tacit knowledge) sowie von sozialem, kulturellem und geographischem Kapital gegenüber. Dies bedeutet eine (neue) Hinwendung zum Raum und insbesondere zu städtischen Räumen, wenn es um die Entwicklung von Gesellschaft geht.

Der Bedeutungszuwachs großstädtischer Ballungsräume schlägt sich derzeit in der Neuorientierung der Raumordnungs- und Raumentwicklungspolitiken vieler europäischer Staaten nieder. Innerhalb der von der Ministerkonferenz für Raumordnung im Sommer 2006 beschlossenen Leitbilder kommt den Metropolregionen eine „Motorenfunktion“ für Wachstum und Innovation sowie für die sozialen und gesellschaftlichen Entwicklungen in Deutschland zu. Regionalentwicklung soll damit stärker als bisher an erkennbaren wirtschaftlichen Kristallisationspunkten ansetzen und darüber „Überschüsse“ produzieren, die im Sinne des Ausgleichszieles der Raumordnungspolitik genutzt werden können.

Die „Konstruktion“ der Metropolregionen folgt bislang nationalen Logiken. Konstitutiv für Metropolräume im 
raumordnungspolitischen Verständnis in Deutschland ist eine Konzentration von Metropolfunktionen insbesondere in den Metropolenkernen, aber auch im engeren metropolitanen Verflechtungsraum. Im Raumordnungsbericht 2005 wurden die Standorte metropolitaner Funktionen auf der Basis eines einheitlichen Indikatorensets festgelegt (BBR 2005: 177 ff.). Darüber hinaus definieren sich die Metropolregionen des Leitbildes „Wachstum und Innovation“ in ihrer Großräumigkeit über einen ,weiteren“ metropolitanen Verflechtungsraum, der auch ländlich strukturierte Räume mit einschließt. Dieser großräumigen Konstruktion liegt die Überlegung zugrunde, dass die Wachstumskerne eine Schlüsselrolle für (über)regionale Kooperationen und Wachstumsbündnisse besitzen: „Der weitere metropolitane Verflechtungsraum deutet die notwendige Entstehung großräumiger Verantwortungsgemeinschaften an, die für eine ausgeglichene Entwicklung aller Raumtypen notwendig sind“ (BMVBS/BBR 2006: 40). Es geht ,langfristig darum, die Zusammenarbeit von starken und schwachen Räumen $\mathrm{zu}$ organisieren, die immer stärker und großräumiger miteinander verflochten und aufeinander angewiesen sind“" (BMVBS/BBR 2006: 58).

Bereits früh wurde festgestellt: „In grenznahen Gebieten liegen teilweise wichtige Metropolfunktionen außerhalb Deutschlands“" (BBR 2005: 185) und mit dem Verweis auf die Oberrheinregion wird die Option eines ,grenzüberschreitenden europäischen Metropolraumes“ in die Diskussion gebracht. Diese Überlegungen fanden allerdings bei der Ausweisung der Metropolregionen zunächst keine Berücksichtigung.

Die elf europäischen Metropolregionen in Deutschland wurden in zwei Phasen - 1997 und 2005 - von der Ministerkonferenz für Raumordnung (MKRO) anerkannt. Die Metropolregionen selbst vollziehen mit ihren Regionalisierungen die raumordnungspolitischen und analytischen Ansätze zwar nach. Letztendlich stellen die Metropolräume jedoch flexible, auf formalen oder informellen Kooperationen basierende Handlungsräume dar. Den räumlichen Abgrenzungen liegen unterschiedliche Governance-Modelle und administrative Verfassungen zugrunde. Diese reichen in Bezug auf den Kristallisationskern der Metropolitan Governance von gesetzlich institutionalisierten regionalen Verbänden wie in der Metropolregion Rhein-Neckar über informelle kommunale Zusammenschlüsse wie in Nürnberg und München bis hin zur gemeinsamen Landesplanung Berlin-Brandenburg (Ludwig/Mandel/Schwieger et al. 2008: 184 f.).

\subsection{Konzepte in den Nachbarstaaten}

Die Konzepte der Nachbarstaaten unterscheiden sich zum Teil sehr deutlich von dem durch die deutsche Raumordnung eingeschlagenen Weg. Gerade wenn es um die Rolle der grenzüberschreitenden Verflechtungsräume geht, scheint es von besonderer Bedeutung, sich mit den Raumordnungspolitiken der Nachbarstaaten - hier an den Beispielen Frankreichs, der Schweiz und Luxemburgs - auseinanderzusetzen.

In Frankreich beschreitet das „Comité interministériel d'aménagement du territoire“ (CIADT) einen vollständig anderen Weg, um die Konkurrenzfähigkeit französischer Metropolen - jenseits der Île de France - im europäischen und internationalen Wettbewerb zu fördern. Die CIADT rief 2004 unter dem Titel „Pour un rayonnement européen des métropoles françaises. Appel à coopération métropolitaine“ einen Wettbewerb aus, der Regionen dazu auffordert, sich mit metropolitanen Kooperationen und Projekten zu bewerben (DATAR 2004). Voraussetzung für die Bewerbung als Metropole war, dass die Agglomeration ${ }^{4}$ mindestens 500.000 Einwohner aufweist sowie mindestens ein urbanes Gebiet mit mehr als 200.000 Einwohnern und mehreren mittelgroßen Städten einschließt (DATAR 2004 8; DIACT 2005a). 2005 wurden 15 Regionen ausgewählt (vgl. Abb. 1), die - mit (finanzieller) Unterstützung der nationalen Ebene - in den nächsten Jahren die metropolitane Kooperation und assoziierte Projekte auf den Weg bringen. Acht der 15 Regionen sind grenzüberschreitend organisiert und beziehen die Nachbarstaaten direkt mit ein, was von Seiten der Mission Opérationnelle Transfrontalière ${ }^{5}$ (MOT 2005: 2) als besonderer Erfolg gewertet worden ist.

In der Schweiz sind Metropolregionen als Handlungsräume in der politischen Arena bislang kaum verankert (BFS/Ernst Basler + Partner 2007: 50). Das „Raumkonzept Schweiz“ (vgl. Rumley 2008), das die Bedeutung der Metropolitanräume hervorhebt, ohne diese funktional oder räumlich näher zu konkretisieren, wurde von der Bundesebene initiiert und im Rahmen eines breit angelegten kooperativen Prozesses mit Akteuren aller Ebenen erarbeitet. Es stellt die jüngste Etappe einer intensiven und oftmals kontroversen Diskussion um die urbane Entwicklung in der Schweiz dar.

Bereits im Jahr 2000 wurden die Agglomerationen der Schweiz grenzüberschreitend bestimmt: Zehn der 50 Agglomerationen beziehen Gebiete anderer Nationalstaaten ein. 1990 wurden erstmals Agglomerationen ,in den Einzugsgebieten der Großzentren zu statistischen Zwecken zusammengefasst" und damals als Metropolitanräume bezeichnet

\footnotetext{
${ }^{4}$ Die französische Agglomerationsdefinition unterscheidet anhand Zentrenstruktur, Pendlerverflechtungen und Einwohnerzahlen die „communauté d'agglomération“ mit mindestens 50.000 Einwohnern und die „communauté urbaine“ mit mindestens 500.000 Einwohnern.

${ }^{5} 1997$ auf die Initiative des französischen Staates hin gegründet, erhält die MOT 1998 die Form eines Vereins als Austausch-Plattform für Grenzregionen. Die MOT vereint Gebietskörperschaften, Akteure des Wirtschafts- und Sozialgeschehens sowie Institutionen, die an der Entwicklung grenzüberschreitender Projekte beteiligt sind, wobei diese aus Frankreich und neun weiteren europäischen Staaten kommen; vgl. http://www.espaces-transfrontaliers.org (letzter Zugriff am 03.06.2009).
} 
Abb. 1 Metropolitane Kooperationsräume in Frankreich. (Kartographie: Planungsgruppe agl auf der Basis von Daten aus DIACT 2005b)

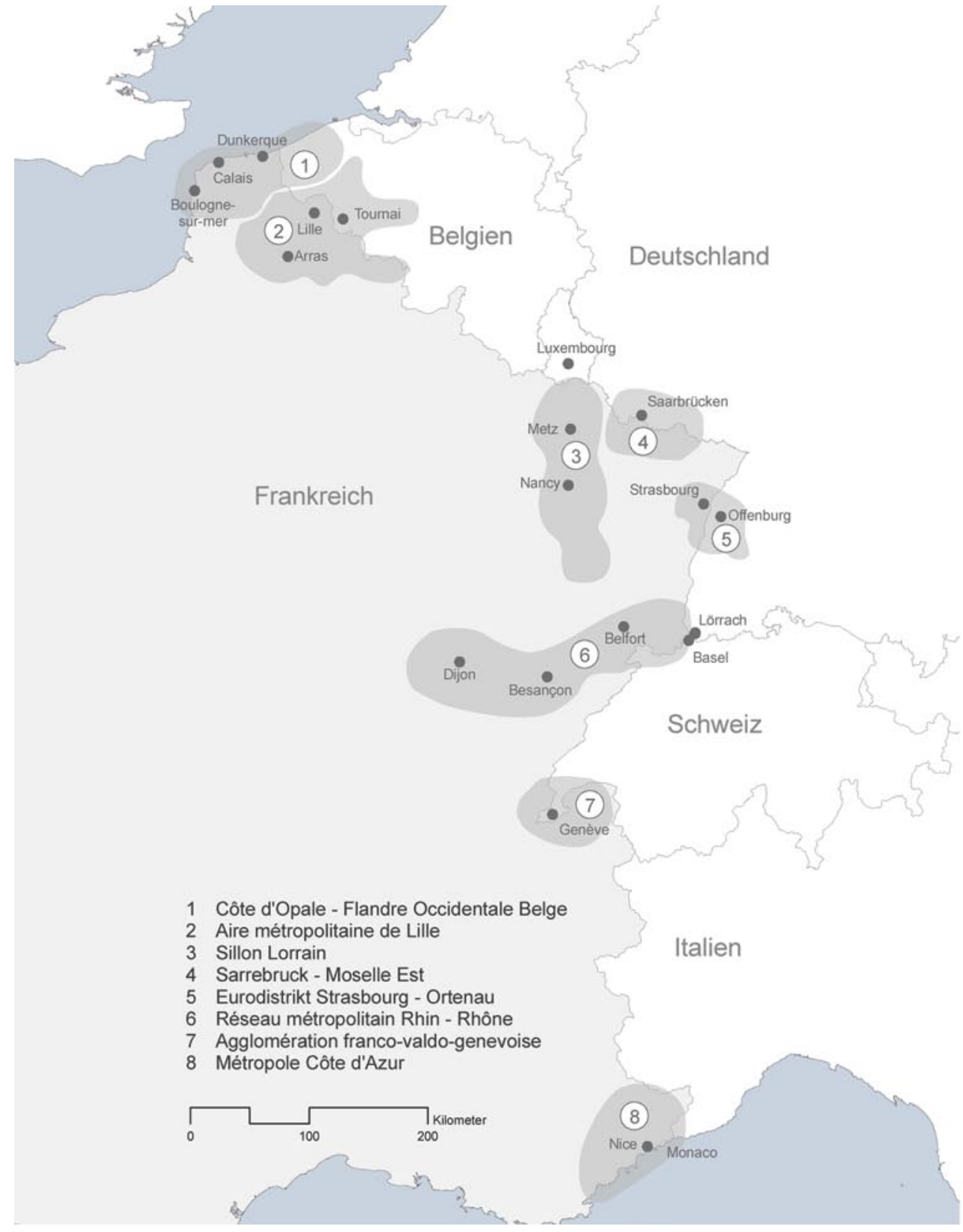

(BFS/Ernst Basler + Partner 2007: 9). Diese wurden 2000 im gleichen Flächenumgriff für die Darstellung der fünf Metropolräume übernommen: „Im europäischen Vergleich wird die heutige Abgrenzung mit den fünf Metropolräumen Zürich, Basel, Genf-Lausanne, Bern und dem Tessin als wenig plausibel eingeschätzt. In der Schweiz werden je nach Standpunkt zwei (Nordschweiz, Arc Lémanique) oder drei Metropolregionen (Zürich, Basel, Arc Lémanique) ausgemacht" (BFS/Ernst Basler + Partner 2007: 51). Die Abgrenzung der Metropolräume bezieht sich ausschließlich auf die Pendlerverflechtungen im Umland bzw. zwischen multipolaren Agglomerationssystemen, wobei auch diese grenzüberschreitend festgelegt wurden (BFS/Ernst Basler + Partner 2007: 10).
In Luxemburg wird derzeit die erste Generation von Landesentwicklungsplänen erarbeitet. Mit den Landesentwicklungsplänen (Plans Sectoriels) in den Bereichen Transport, Wohnen, Gewerbe und Landschaft entsteht ein rechtsverbindlicher und koordinierter Planungsrahmen für das gesamte Luxemburg. Planerische Grundlagen sind das „Programme Directeur d'Aménagement du Territoire“ (Ministère de l'Intérieur, Direction de l'Aménagement du Territoire et de l'Urbanisme 2003) und das Integrative Verkehrs- und Landesentwicklungskonzept (Le Gouvernement du Grand-Duché de Luxembourg 2004). Beide folgen dem Leitbild der dezentralen Konzentration und polyzentrischen Entwicklung. Es werden drei zentrale Aktionsräume im „milieu urbain“ definiert: Luxemburg-Stadt (espace à struc- 
turer), Süd-Agglomeration (espace à régénérer), Nordstad (espace à développer). Das „Programme Directeur“ setzt sich zudem mit der Rolle der Grenzregion im Zusammenspiel der Metropolregionen Nordwesteuropas auseinander und will die Kooperation der Städte in der Grenzregion stärken.

\subsection{Vergleichbare Zielsetzungen - unterschiedliche (räumliche) Implementierung}

Wenngleich die nationalen Raumordnungspolitiken mit der Ausweisung oder Initiierung von Metropolregionen vergleichbare Ziele und strategische Ansätze verfolgen (BAK Basel economics 2006: 9), so zeigen sich doch grundsätzliche Unterschiede im „Konstruktionsprozess“ sowie der räumlichen und akteursbezogenen Implementierung. Die neuen Leitbilder der Raumordnung in Deutschland unterscheiden sich in Bezug auf die großräumige „Konstruktion“ der Metropolregionen, die auf eine überregionale Integration von Wachstumskernen und eine Stabilisierung der Regionalisierungsprozesse durch überregionale Partnerschaften abzielt, deutlich von raumordnungspolitischen Ansätzen in Frankreich, der Schweiz und in Luxemburg.

Zudem zeigen sich Unterschiede in der Einordnung grenzüberschreitender Verflechtungsräume. Im Gegensatz zu Frankreich und der Schweiz finden die grenzüberschreitenden Verflechtungen in der bundesdeutschen Raumordnung (noch) keine angemessene Berücksichtigung. Gerade im Leitbild 1 „Wachstum und Innovation“, in dem den Metropolregionen im Sinne der Lissabon-Strategie eine Schlüsselrolle zugesprochen wird, bleibt der räumliche Ansatz eher binnen- und weniger europäisch orientiert. Zwar sollen die Metropolen als Wachstumspole über die Bildung großräumiger Verantwortungsgemeinschaften die umliegenden Regionen bis in den eher ländlich strukturierten und peripheren Raum hinein einbinden. Diese weiteren funktionalen Verflechtungsräume bilden jedoch keine grenzüberschreitenden Verflechtungen ab. Demgegenüber lassen die Raumentwicklungsstrategien von Frankreich und der Schweiz zwar erkennen, dass grenzüberschreitende Verflechtungsräume Eingang in die politischen Überlegungen zur Neuausrichtung des raumordnungspolitischen Systems gefunden haben. Allerdings bleiben diese Ansätze auf die engeren Umgriffe der grenzüberschreitenden Agglomerationen beschränkt und beziehen sich nicht auf großräumige grenzüberschreitende Verflechtungsräume.

\section{Spezifika großräumiger grenzüberschreitender Verflechtungsräume}

Was zeichnet grenzüberschreitende Verflechtungsräume aus? Ausgangspunkt ist die Überlegung, dass ihnen vor dem
Hintergrund des Leitbildes „Wachstum und Innovation“ durchaus eine besondere Rolle auf nationaler und europäischer Ebene zukommt. Diese Rolle bezieht sich sowohl auf die Möglichkeit, Wachstum und Innovation zu erzeugen, als auch auf das strategische Potenzial dieser Grenzregionen für den europäischen Integrationsprozess. Um diese Rolle ausfüllen und das notwendige „Gewicht“ in die raumordnungspolitische Diskussion einbringen zu können, stehen hier großräumige Regionalisierungsprozesse im Vordergrund. Vor diesem Hintergrund lassen sich in einer ersten Annäherung konstitutive Merkmale grenzüberschreitender Verflechtungsräume herausstellen, die diese von nationalen Binnenregionen, aber auch von anderen Grenzregionen differenzieren.

\subsection{Intensive grenzüberschreitende funktionale Verflechtungen}

Wie lassen sich intensive grenzüberschreitende Verflechtungen nachweisen? Die Verfügbarkeit vergleichbarer Daten auf Gemeinde- oder NUTS 3-Ebene zum Nachweis grenzüberschreitender Verflechtungen erweist sich als außerordentlich schwierig und lückenhaft (BMVBS 2010: 8). In den untersuchten MORO-Regionen lassen sich funktionale Verflechtungen für die grenznahen Bereiche gut nachvollziehen; großräumige Kooperationsräume wie beispielsweise die Großregion SaarLorLux lassen sich hierdurch analytisch allerdings nicht belegen (BMVBS 2010: 9).

Ein „Motor“ für grenzüberschreitende Verflechtungen und für den funktionalen Integrationsprozess ist das meist erhebliche Strukturgefälle, das sich entlang nationaler Grenzen bei der Betrachtung einzelner Parameter wie beispielsweise des Lohn-, Kaufkraft-, Preis- oder Bodenpreisgefälles zeigt. Großräumige Zuschnitte wie bei den untersuchten MORORegionen schließen zudem grenzüberschreitende Agglomerationen oder Städtenetze ein, in denen die Bevölkerung das vielfältige Angebot an Arbeitsplätzen und Dienstleistungen, Bildungsstätten, kultureller Aktivität und Freizeiteinrichtungen beidseits der Grenzen nutzt; dazu gehören auch speziell auf Grenzräume ausgelegte Infrastrukturen und Angebote wie beispielsweise bilinguale Kindergärten, Schulen, Ausbildungsstätten und Studiengänge.

\subsection{Etablierte institutionelle Arrangements grenzüberschreitender Zusammenarbeit}

Anders als bei den (meisten) Metropolregionen in Deutschland existieren in vielen Grenzregionen bereits funktionierende institutionelle Arrangements großräumigen Zuschnitts mit einer langen Tradition in der grenzüberschreitenden Kooperation. Da hierarchische Steuerungsoptionen und formale Sanktionierungsmöglichkeiten im grenzüberschreitenden Kontext weitgehend fehlen, haben 
sich in etablierten Grenzregionen vielfältige Kooperationsstrukturen und -kompetenzen auf allen Ebenen herausgebildet. Hierzu zählen unterschiedliche Organisationsformen und Netzwerke ebenso wie institutionelle und zivilgesellschaftliche Routinen im Umgang mit der „Grenze“. Demgemäß sind die entstandenen Governance-Strukturen und Praktiken konstituierend für Grenzregionen. Sie funktionieren unter spezifischen und oft schwierigen Rahmenbedingungen: kulturellen Unterschieden und Sprachbarrieren, dem Aufeinandertreffen unterschiedlicher Rechts-, Politik-, Verwaltungs- und Planungssysteme sowie institutioneller Asymmetrien, (bislang) fehlender transnationaler Rechtsinstrumente und unmittelbarer politischer Legitimationsverfahren (vgl. hierzu Knippschild 2009; Beck 2010). Grenzregionen sind auf ein freiwilliges Aushandeln von Interessen, eine konsensorientierte Zusammenarbeit und die Umsetzung erzielter Konsense in den jeweiligen nationalen Systemen angewiesen. Deshalb liegt in den Erfahrungen mit grenzüberschreitender Kooperation eine Schlüsselkom- petenz begründet, die Grenzregionen für eine erfolgreiche transnationale Zusammenarbeit in Europa befähigt.

\subsection{Ausbildung großräumiger Kooperationsräume}

Vergleichbar zu den Metropolregionen in Deutschland geht es bei den grenzüberschreitenden Verflechtungsräumen um großräumige regionale Zuschnitte und nicht um einzelne Stadt- oder Arbeitsmarktregionen, kleinere Agglomerationen oder Eurodistrikte. Ein Blick auf die Flächenkulisse der Euregio Maas-Rhein, der Großregion SaarLorLux + , der Oberrhein- und der Bodenseeregion zeigt, dass es sich um polyzentrische Regionen handelt, die eine hoch differenzierte Raumstruktur aufweisen (vgl. exemplarisch Abb. 2). Sie umfassen unterschiedlichste Raumtypen - neben Agglomerationen und Stadtregionen auch periphere, ländlich strukturierte Räume. Die Kernstädte der Regionen übernehmen oberzentrale Funktionen für ihre Verflechtungsbereiche, die häufig über nationalstaatliche Grenzen hinweg



Abb. 2 Städtesystem der Großregion Saar-Lor-Lux+. (Kartographie: Planungsgruppe agl auf der Basis von Geo-Daten des Bundesinstituts für Bau-, Stadt- und Raumforschung und der Regionen) 
ausstrahlen und teilweise bereits in grenzüberschreitenden Städtenetzen organisiert sind.

\subsection{Potenziale für Wachstum und Innovation}

Bereits in der ersten Abfassung der neuen Leitbilder des Bundes und der Länder wurde darauf verwiesen, dass es auch „Wachstumsmotoren außerhalb der Metropolregionen“ gibt (BMVBS/BBR 2006: 42). Die grenzüberschreitenden Verflechtungsräume zeichnen sich durch zukunftsfähige Wirtschaftsstandorte und Wachstumspotenziale aus. Diese sind nicht gleichmäßig über die nationalen Teilräume verteilt; vielmehr gibt es deutliche Schwerpunkte wie beispielsweise Luxemburg-Stadt im Bereich der Großregion SaarLorLux mit einem selbst für gesamteuropäische Verhältnisse außergewöhnlichen Wirtschaftswachstum. Die gleiche Situation ergibt sich auch bei der Analyse der Metropolfunktionen (BBR 2005: 185): Es zeigt sich, dass Grenzregionen über metropolitane Teilfunktionen verfügen - durchaus vergleichbar mit einigen der ausgewiesenen Metropolregionen in Deutschland. Allerdings gilt auch hier, dass die metropolitanen Funktionen ebenso wie die Wachstumspotenziale in den nationalen Teilräumen aufgrund der Barrierewirkung nationalstaatlicher Grenzen weitaus weniger stark für die gesamte Grenzregion wirksam werden.

\section{Das Modellvorhaben der Raumordnung „Überregionale Partnerschaften in grenzüberschreitenden Verflechtungsräumen“"}

\subsection{Die politische Initiative zur Rolle der grenzüberschreitenden Verflechtungsräume in der deutschen Raumordnung}

Eine vom Bundesamt für Bauwesen und Raumordnung, der Akademie für Raumforschung und Landesplanung und dem Regionalverband Bodensee-Oberschwaben durchgeführte Fachveranstaltung kam bereits $2005 \mathrm{zu}$ dem Ergebnis, dass sich „deutliche Hinweise darauf ergeben, dass gerade bei grenzüberschreitenden Wachstumsräumen, vielleicht aber nicht nur bei diesen, das Konzept der Metropolregionen um andere Wachstumsmotoren zu ergänzen ist" (Köhler 2007: 118). Als Beispiele dienten die Regionen Bodensee-Oberschwaben und Regensburg/Oberpfalz sowie die schweizerische Region Chur/Bündner Rheintal und die österreichische Region Linz/Oberösterreich. So hat sich die Region Bodensee-Oberschwaben in den vergangenen Jahren zu einer der dynamischsten und erfolgreichsten Regionen innerhalb von Deutschland entwickelt, wobei gerade die Arbeitsmarktsituation - wie auch in anderen Teilräumen rund um den Bodensee - für europäische Verhältnisse außergewöhnlich gut ist (vgl. auch Köhler 2009).
Die Arbeitsgruppe des Beirats für Raumordnung zur Weiterentwicklung des Leitbilds „Wachstum und Innovation" regte bereits ein Jahr nach dem Bestehen der Leitbilder gegenüber dem Bundesministerium für Verkehr, Bau- und Stadtentwicklung (BMVBS) an, ,auf eine Anerkennung bzw. eine begleitende Unterstützung in der Initiierung und Etablierung eines Initiativkreises Europäische Verflechtungsräume in Deutschland" hinzuwirken. ${ }^{6}$ Weiter wurde dem Ministerium „eine verstärkte Ausrichtung des MORO-Programmes des BMVBS auf Kooperationsstrategien innerhalb von Verantwortungsgemeinschaften von Metropolregionen bzw. europäischen Verflechtungsräumen, auf Vernetzungsstrategien von Metropolregionen und Europäischen Verflechtungsräumen unter- wie auch miteinander sowie auf die Initiierung Europäischer Verflechtungsräume" empfohlen. ${ }^{7}$ Damit verbindet sich implizit auch die Forderung nach einer Europäisierung der nationalen Raumordnungspolitik und einer stärkeren Berücksichtigung des Zieles der territorialen Kohäsion für Europa.

Das Bundesministerium für Verkehr, Bau und Stadtentwicklung und das Bundesinstitut für Bau-, Stadt- und Raumforschung haben diese Anregungen aufgegriffen. Im Rahmen der so genannten Modellvorhaben der Raumordnung (MORO) unterstützt der Bund die gemeinsame Initiative der Regio Aachen, der Regionalverbände Mittlerer Oberrhein, Bodensee-Oberschwaben, Hochrhein-Bodensee und Südlicher Oberrhein sowie des Saarlandes, die Rolle der grenzüberschreitenden Verflechtungsräume im Zusammenspiel mit den europäischen Metropol- und Städteregionen und vor dem Hintergrund der Weiterentwicklung der Leitbilder in Deutschland näher zu beleuchten.

Die regionalen Partner des Modellvorhabens vertreten Institutionen innerhalb der Euregio Maas-Rhein, der (europäischen) Großregion (SaarLorLux+), der Trinationalen Metropolregion Oberrhein sowie der Bodenseeregion. Die organisatorische Federführung des Projektes mit einer Laufzeit von Oktober 2008 bis Ende 2010 hat das Saarland, vertreten durch das Ministerium für Umwelt, Energie und Verkehr, übernommen.

Das Modellvorhaben steht im Kontext des MORO-Forschungsfeldes „Überregionale Partnerschaften“, dessen Ziel es ist, den strategischen Ansatz der ,großräumigen Verantwortungsgemeinschaften“ des Leitbildes „Wachstum und Innovation" der Raumordnung des Bundes und der Länder in der Umsetzung zu erproben. „Großräumige Verantwortungs-

\footnotetext{
${ }^{6}$ Empfehlung der Arbeitsgruppe „Entwicklungsgedanken“ im Beirat für Raumordnung zur Weiterentwicklung des Leitbildes „Wachstum und Innovation", verabschiedet auf der Sitzung des Beirats für Raumordnung am 20.09.2007.

${ }^{7}$ Empfehlung der Arbeitsgruppe „Entwicklungsgedanken“ im Beirat für Raumordnung zur Weiterentwicklung des Leitbildes „Wachstum und Innovation“, verabschiedet auf der Sitzung des Beirats für Raumordnung am 20.09.2007.
} 
gemeinschaften" sollen als innovatives Instrument einer auf Entwicklung und Ausgleich ausgerichteten Raumordnungspolitik zwischen Städten, Metropolen und ländlichen Wachstumsregionen sowie peripheren und strukturschwachen Regionen implementiert werden. „In einem partnerschaftlichen Miteinander dieser strukturell und ökonomisch unterschiedlichen Regionstypen sollen alle Teilräume dazu beitragen, dass insbesondere Wachstum und Innovationen gestärkt werden. Damit verbindet sich das Bestreben, alle Räume - die starken und die schwachen - zu befähigen, ihre Potenziale zu erkennen, zu bündeln und zu vernetzen“" (BBR 2008: 4). 2007 startete hierzu das Modellvorhaben „Überregionale Partnerschaften - Innovative Projekte zur stadtregionalen Kooperation, Vernetzung und gemeinsamen großräumigen Verantwortung" mit sieben Modellregionen, das 2008 auf die grenzüberschreitenden Verflechtungsräume ausgeweitet wurde (BBSR 2009: 5; vgl. Abb. 3).

\subsection{Ziele des MORO und der Projektpartnerschaft}

Die MORO-Partnerschaft sieht sich in der Verantwortung, einen Beitrag zur Weiterentwicklung der räumlichen Leitbilder des Bundes und der Länder hinsichtlich der Rolle grenzüberschreitender Verflechtungsräume zu leisten. Gleichzeitig bewegt sie sich innerhalb des europäischen Mehrebenensystems. Neben der nationalstaatlichen Ebene wird aufgrund der grenzüberschreitenden Dimension unmittelbar die europäische Ebene adressiert, zumal sich die Regionen im Zuge der Regionalisierung der europäischen Strukturpolitik seit Mitte der 1990er Jahre als wichtige Ebene europäischer Politik etablieren konnten (Staeck 1997: 38; Schmitt-Egner 2005: 29 f.). Ziel des Modellvorhabens ist es deshalb, die großräumigen grenzüberschreitenden Verflechtungsräume im Zusammenspiel der europäischen Stadt- und Metropolregionen sowohl auf nationaler wie auch auf europäischer Ebene zu profilieren (Hartz 2009) und hierzu Anforderungen und Empfehlungen an die Raumordnungs- und Raumentwicklungspolitiken sowie an raumrelevante Fachpolitiken auf nationalstaatlicher und europäischer Ebene zu formulieren, um die raumordnungspolitischen Rahmenbedingungen für die Grenzregionen zu verbessern.

Zudem richtet sich das Interesse der MORO-Partnerschaft auf die Regionen selbst. Das Projekt soll auch nach ,innen“ wirksam werden. Im Rahmen des MORO sollen gemeinsame Projektinitiativen auf den Weg gebracht werden, die eine kohärente und nachhaltige grenzüberschreitende Regionalentwicklung sowie effektive Kooperations- und Governance-Strukturen unterstützen und damit den inneren Zusammenhalt und die regionale Integration über nationale Grenzen hinweg stärken. Die MORO-Partnerschaft verfolgt darüber hinaus die Gründung eines Initiativkreises als offenes Bündnis der grenzüberschreitenden Verflechtungsräume - mit dem Ziel, eine langfristige und tragfähige Part- nerschaft zu institutionalisieren und anderen Grenzregionen die Möglichkeit zur Mitarbeit zu eröffnen. Die Verstetigung der Netzwerkarbeit wird als dauerhafter Prozess über die Förderperiode des MORO hinaus verstanden.

\subsection{Forschungs- und Handlungsfelder}

\subsubsection{Gemeinsames Selbstverständnis der grenzüberschreitenden Verflechtungsräume entwickeln}

Eine Voraussetzung für die erfolgreiche Etablierung grenzüberschreitender Verflechtungsräume im Kontext raumordnerischer Konzepte ist ein gemeinsames Verständnis über deren konstituierende Merkmale. Von dieser Begriffsklärung hängen nicht nur Resonanz und Akzeptanz in der raumordnungspolitischen Diskussion, sondern auch die Wahrnehmung der grenzüberschreitenden Verflechtungsräume als wichtiges regionalpolitisches Handlungsfeld ab. In der Innenwirkung geht es darum, regionale grenzüberschreitende Identität(en) oder zumindest die Wahrnehmung der Grenzregion als Basis für ein Region Building sowie für die Unterstützung institutioneller Arrangements zu stärken. Ein gemeinsames Selbstverständnis ist zudem eine unverzichtbare Grundlage für eine zielgerichtete und effiziente Partnerschaft.

In diesem Zusammenhang stellt sich die Frage, wie grenzüberschreitende Verflechtungsräume definiert werden können: Was zeichnet diese Regionen gegenüber anderen Grenzregionen oder Regionen innerhalb der Nationalstaaten aus? Die bisherigen Ergebnisse im Rahmen des Modellvorhabens lassen sich auf die in Kap. 4 genannten Merkmale zuspitzen. Diese sollen weiter konkretisiert und - soweit möglich - auf der Grundlage von Indikatoren und Kriterien empirisch belastbar hinterlegt werden.

Im Vordergrund stehen diejenigen Indikatoren, welche die für Grenzregionen typischen Verflechtungen und Integrationsprozesse über nationale Grenzen hinweg abbilden. Von besonderem Interesse sind beispielsweise Pendlerverflechtungen in den Sektoren Arbeit und Ausbildung, Wohnen und Freizeit oder grenzüberschreitende Unternehmenskooperationen und Wertschöpfungsketten. Im Fokus stehen zudem Angebote und Infrastrukturen, die speziell auf Grenzräume ausgelegt sind, wie z. B. bilinguale Ausbildungsstätten und Studiengänge. Über diese Indikatoren können raumstrukturelle und sozioökonomische Verflechtungen aufgezeigt und gleichzeitig der „Mehrwert“ der großräumigen grenzüberschreitenden Kooperation sichtbar gemacht werden.

Grundsätzlich stellt die territoriale Abgrenzung ein Problem dar, das alle großräumigen Regionalisierungen begleitet. Diese Abgrenzung kann indikatorenbasiert vorgenommen werden und somit die Region als Analysekategorie abbilden. Aus politischer Perspektive stellen Regionen 


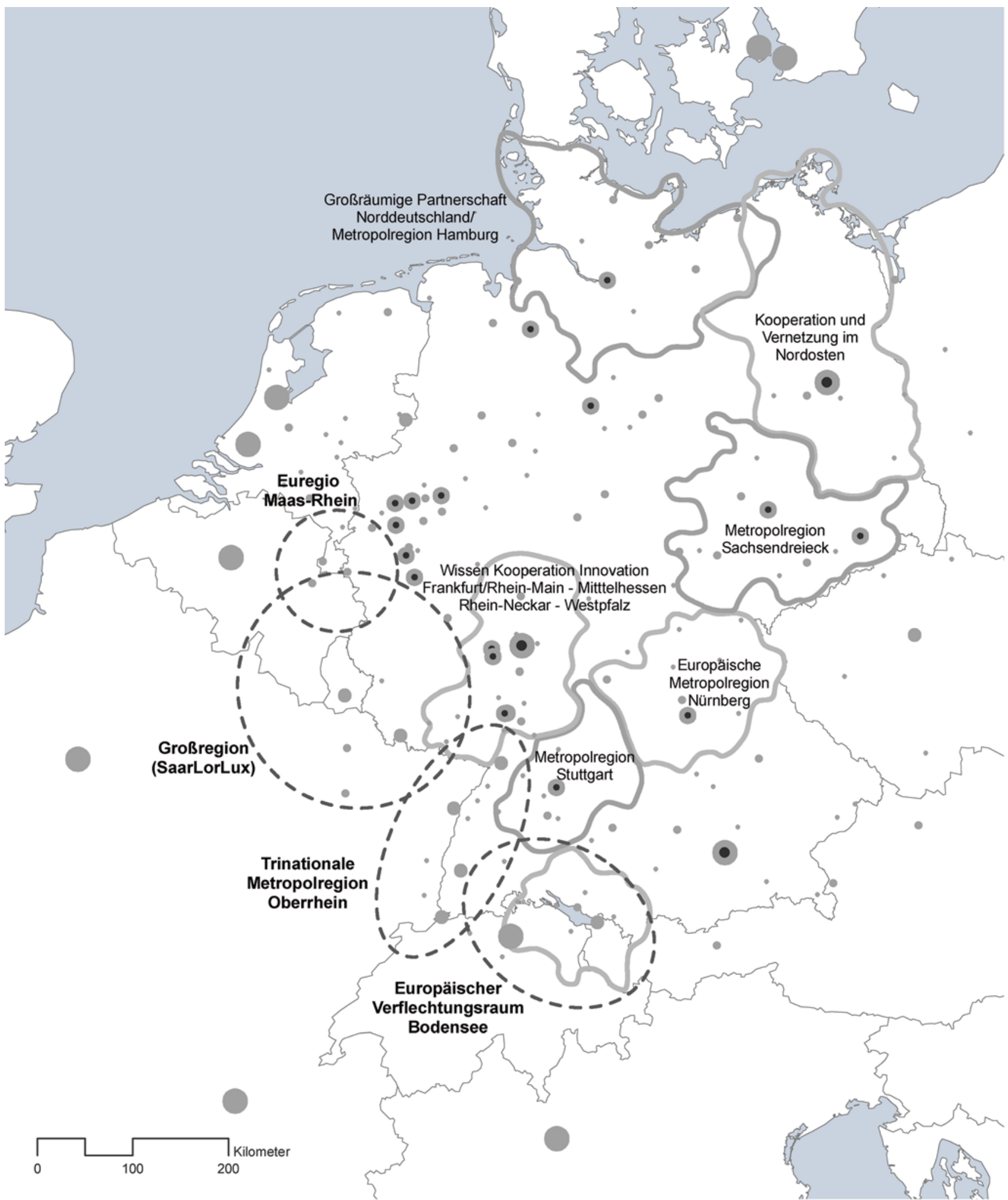

I _ _ I Grenzüberschreitende Verflechtungsräume (MORO) MORO-Modellregionen "Überregionale Partnerschaften"

Kerne der bestehenden Metropolregionen

Abb. 3 Die MORO-Projektfamilie (Kartographie: Planungsgruppe agl auf der Basis von Geo-Daten des BBSR) 
kooperative Handlungsräume von Akteuren (Blotevogel/ Schulze 2009) dar, durchaus - in Abhängigkeit von Kooperations- und Institutionalisierungsprozessen - mit variablen Geographien. Das Aufzeigen der räumlichen Dimension intensiver funktionaler Verflechtungen in Grenzräumen kann hier einen Beitrag zur Diskussion des „Zuschnitts“ grenzüberschreitender Handlungsräume sowie einer effektiven „Arbeitsteilung“ der nationalen Teilräume leisten.

\subsubsection{Stärken stärken - Hemmnisse abbauen}

Die Standortnachteile von Grenzregionen liegen - trotz Schengener Abkommen und regionalpolitischer Initiativen wie INTERREG - auf der Hand: Nationalstaatliche Grenzen wirken immer noch als Barrieren mit weit reichenden Konsequenzen für eine kohärente Regionalentwicklung: beispielsweise das Auseinanderfallen funktionaler und territorial-administrativer Strukturen, Unterschiede in den Planungs- und Verwaltungskulturen sowie Doppelstrukturen in den nationalen Teilräumen, die einer Bündelung der Kräfte in der Region entgegenstehen. Hinzu kommen Informationsdefizite und nach wie vor wirksame Stereotype im Umgang miteinander.

Grenzüberschreitende Verflechtungsräume präsentieren sich auf der anderen Seite als außerordentlich vielfältige und europäisch orientierte gemeinsame Lebens-, Wirtschaftsund Kulturräume. Sie besitzen ein erhebliches Wachstumsund Innovationspotenzial und zugleich ein strategisches Potenzial für den europäischen Integrationsprozess.

Integrierte grenzüberschreitende Raumentwicklungskonzepte können diese strategische Ausrichtung unterstützen und das Handeln der beteiligten Akteure auf ein gemeinsames Bündel hinreichend konkreter Ziele und Maßnahmen zur Entwicklung der Grenzregion ausrichten. Damit können die spezifischen Standortvorteile und die grenzüberschreitende (kulturelle) Vielfalt besser ausgeschöpft, gleichzeitig grundsätzliche Wandlungsprozesse der Gesellschaft wie auch aktuelle Trends und Sektorpolitiken wirkungsvoller eingebettet werden. Eine kohärente Raumentwicklung sollte nach „,innen“ und nach ,außen“ konkretisiert werden. Das ,innere Funktionieren' der Regionen kann durch eine Optimierung der spezifischen harten und weichen Standortfaktoren sowie deren Zusammenspiel in der Grenzregion verbessert werden. Hierzu zählen sowohl der Ausbau von Infrastrukturen und die Verbesserung der Lebensqualität als auch eine potenzielle Funktionsteilung zwischen den unterschiedlichen Raumtypen sowie den nationalen Teilräumen innerhalb der Grenzregionen.

Zur Stärkung der Rolle und Wettbewerbsfähigkeit grenzüberschreitender Verflechtungsräume müssen konkrete Kooperationsinteressen auf der Basis vorhandener Schwächen und Hemmnisse sowie Stärken und Potenziale identifiziert und als gemeinsame Handlungsfelder etabliert werden. Diese Kooperationsinteressen sollen dabei die großräumigen Regionalisierungsprozesse und strategischen Ziele unterstützen, die sich für die grenzüberschreitenden Verflechtungsräume ergeben.

\subsubsection{Europa im Kleinen: grenzüberschreitende Governance optimieren}

Eine Schlüsselkompetenz der Grenzregionen liegt in den Erfahrungen mit grenzüberschreitender Kooperation begründet. Daraus haben sich vielfältige Strukturen und Netzwerke entwickelt, die teilweise in konkurrierenden räumlichen und fachlichen Zuschnitten sowie in komplexen Überlagerungen der Zuständigkeiten mündeten. In der Praxis zeigt sich dennoch, dass die ausgeprägte Mehrebenen-Problematik in den Grenzregionen und hochkomplexe grenzüberschreitende Fragestellungen vergleichsweise unterkomplexen Strukturen und nationalstaatlich organisierten Regelungsmechanismen gegenüberstehen. ${ }^{8}$ Die grenzüberschreitende Zusammenarbeit ist zudem meist inter-institutionell und nicht intermediär ausgerichtet. Der Abbau regionalökonomisch, sozial und kulturell bedingter Stereotype gehört zwar zur Praxis der transnationalen Kooperation, dennoch zeigen sich diese Strukturen als außerordentlich persistent und meist wenig reflektiert auf der Handlungsebene. Sie schränken die Wettbewerbs- und Handlungsfähigkeit sowie die Kommunikation über die Grenzen hinweg deutlich ein und wirken meist „im Verborgenen“.

Der Ausbildung effektiver Governance-Strukturen in grenzüberschreitenden Verflechtungsräumen muss im Rahmen nationaler und europäischer Politiken und Förderprogramme stärkeres Gewicht beigemessen werden. Die Grenzregionen sollen in ihrem Bemühen um eine „Europäisierung" institutionellen Handelns sowie in ihrer Suche nach vorbildlichen und zukunftsweisenden Strategien zur Forcierung des europäischen wie regionalen Integrationsprozesses adäquat unterstützt werden („Europa im Kleinen“).

$\mathrm{Zu}$ grenzüberschreitenden Governance-Strukturen, die in aller Regel auf Einstimmigkeit und Konsens basieren, müssen auch Strategien zur Bewältigung „harter“ Interessenskonflikte gehören. In der Bearbeitung und Lösung widersprüchlicher Interessen liegt ein grundsätzlicher Schwachpunkt der grenzüberschreitenden Kooperation (ÖROK 2005: 21). So weisen Scherer und Schnell (2002: 517) darauf hin, dass die grenzüberschreitende Kooperation in der Regio Bodensee als „Schönwetter-Politik“ verstanden wird, da innerhalb der grenzüberschreitenden Gremien

\footnotetext{
${ }^{8}$ Zwischenergebnisse der Expertise „Kooperations- und Governancestrukturen in grenzüberschreitenden Verflechtungsräumen. Analyse der bestehenden grenzüberschreitenden Strukturen der Zusammenarbeit auf unterschiedlichen Ebenen und räumlichen Zuschnitten" des EURO-Instituts (Kehl), November 2009.
} 
(fast) ausschließlich Themen behandelt werden, bei denen keine Konflikte zwischen den einzelnen Ländern bestehen.

Eine stärkere Einbeziehung von Wirtschafts- und Sozialpartnern sowie weiterer zivilgesellschaftlicher Akteure ist eine weitere zentrale Aufgabe, die ohnedies von den meisten Politiken und Förderprogrammen eingefordert wird. Hier müssen Schnittstellen zwischen Verwaltungshandeln und anderen gesellschaftlichen Sphären aktiv geschaffen werden. Dies gilt gleichermaßen für eine Aktivierung und Beteiligung der Bevölkerung in Fragen der grenzüberschreitenden Zusammenarbeit.

Eine Kernfrage des Modellvorhabens bezieht sich auf die Ausgestaltung großräumiger Verantwortungsgemeinschaften im grenzüberschreitenden Kontext: Wie können großräumige Regionalisierungsprozesse und überregionale Partnerschaften über nationale Grenzen hinweg wirksam unterstützt bzw. initiiert werden, zumal sowohl Handlungsdruck als auch institutioneller Mehrwert hier nicht immer auf der Hand liegen. Ein entscheidender Schritt kann die Identifikation von Kooperationsinteressen sein, die das Konzept großräumiger Verantwortungsgemeinschaften mit Leben füllen und Schlüsselaufgaben für diese Ebene - im Zusammenspiel mit anderen, kleinräumiger organisierten Handlungsräumen wie beispielsweise den Eurodistrikten - darstellen.

\subsubsection{Initiativkreis grenzüberschreitender Verflechtungsräume: im Netzwerk handeln}

Das Modellvorhaben soll in der Gründung eines Netzwerkes oder Initiativkreises der grenzüberschreitenden Verflechtungsräume münden. Wie erfolgreich die gemeinsame Arbeit sein kann, zeigt der Initiativkreis der europäischen Metropolregionen in Deutschland (IKM). Er bietet eine Plattform für Vernetzung, Austausch und Information. Der Initiativkreis und seine Mitglieder beteiligen sich kontinuierlich und wirkungsvoll an den raumwissenschaftlichen und raumentwicklungspolitischen Diskussionen und profilieren sich damit sowohl auf nationaler als auch auf europäischer Ebene.

Die Ausgestaltung eines Netzwerks der grenzüberschreitenden Verflechtungsräume kann sich (in einem ersten Schritt) auf die Teilräume der Grenzregionen in Deutschland beziehen und sich damit auf eine Wirksamkeit im nationalen Kontext richten. Das Bündnis integriert damit die bundesdeutschen Initiativen, kann diese - vergleichbar dem IKM - außenwirksam vertreten und als Ansprechpartner für europäische Institutionen, Netzwerke und Partner dienen. Eine frühzeitige und aktive Beteiligung der Partner aus den Nachbarstaaten ist hierbei Voraussetzung, um sich auch in einem nächsten Schritt für ein Bündnis ,starker“ Grenzregionen auf einer europäischen Plattform zu engagieren.

\section{Perspektiven für das künftige Europa}

Das „Grünbuch zum territorialen Zusammenhalt“ (Kommission der europäischen Gemeinschaft 2008) widmet sich explizit dem Anliegen eines Zusammenwachsens von Regionen über nationalstaatliche Grenzen hinweg. Das Grünbuch betont die besondere Rolle dieser Regionen für die territoriale Kohäsion in Europa, zeigt aber auch auf, dass gerade diese Regionen aufgrund der schwierigen Rahmenbedingungen oftmals Unterstützung bei dieser Aufgabe brauchen. So wird festgestellt, dass eine verstärkte Zusammenarbeit über regionale und nationale Grenzen hinweg Governance-Fragen aufwirft, beispielsweise die Frage nach neuen legislativen und administrativen Instrumenten, um die Zusammenarbeit, auch entlang der Außengrenzen, zu vereinfachen. Dabei spielt die Arbeitsteilung zwischen den Teilräumen und insbesondere zwischen unterschiedlichen Ebenen der grenzüberschreitenden Kooperation, die über verschiedene institutionelle Arrangements und territoriale Zuschnitte abgebildet werden, eine entscheidende Rolle (BMVBS 2010: 19). Der Fokus des Modellvorhabens der Raumordnung liegt hierbei auf dem Mehrwert, den großräumige Kooperationen im Sinne des Konzeptes der überregionalen Partnerschaften erbringen.

Grenzregionen sind Experimentierräume für eine Stärkung des territorialen Zusammenhalts und eine Intensivierung der Kohäsionspolitik auf europäischer Ebene. Sie können durch spezifische Infrastrukturen, Angebote und Netzwerke eine Gateway-Funktion für nationale Kontexte wahrnehmen. Die MORO-Regionen besitzen zudem spezifische Entwicklungspotenziale für Wachstum und Innovation und können zur erfolgreichen Realisierung des Lissabon-Zieles unter Berücksichtigung der Grundsätze der Nachhaltigkeit (Göteborg-Ziel) beitragen. Um diese Potenziale besser mobilisieren zu können, will das Modellvorhaben zentrale Handlungsfelder in der grenzüberschreitenden Regionalentwicklung aufzeigen (BMVBS 2010: 6). Strategische Handlungsfelder, die eine kohärente Raumentwicklung und die Wettbewerbsfähigkeit der grenzüberschreitenden Verflechtungsräume befördern, beziehen sich $\mathrm{u}$. a. auf eine gemeinsame Geodatenbasis für die Grenzregion oder auch auf eine gemeinsame Sichtweise und Haltung zur Grenzregion - als Basis für ein geteiltes Selbstverständnis und kooperatives Handeln. Operative Handlungsfelder wie beispielsweise die Förderung der grenzüberschreitenden Mobilität oder eine effektivere Vernetzung der Infrastrukturen zur Daseinsvorsorge können durch Aktionsprogramme oder Projektfamilien umgesetzt werden (BMVBS 2010: 6).

Den raumpolitischen Akteuren kommt hierbei eine zentrale Rolle zu. Das Ziel des territorialen Zusammenhalts und damit der territorialen Integration ,,verpflichtet die raumpolitischen Akteure in Europa auf eine horizontal wie vertikal kohärente Ausübung ihrer raumrelevanten Kompetenzen“ 
(Battis/Kersten 2008: 11). Diese Aussage legt nahe, dass gerade die Raumordnung mit ihrem Koordinationsanspruch im grenzüberschreitenden Kontext eine wichtige Rolle übernehmen sollte. Das Modellvorhaben der Raumordnung wird kritisch prüfen müssen, inwieweit die Raumordnung diesem Anspruch bisher gerecht wird und welche Optionen sie hat, ihre Position zu stärken und ihre Kompetenzen erfolgreich in die grenzüberschreitende Kooperation einzubringen.

Die Projektpartnerschaft will einen Beitrag zur Positionierung der grenzüberschreitenden Verflechtungsräume als strategischer Raumtyp leisten. Das Modellvorhaben zielt damit auf eine adäquate Berücksichtigung der Grenzregionen in der deutschen Raumordnungspolitik, insbesondere bei der Weiterentwicklung der raumordnungspolitischen Leitbilder des Bundes und der Länder sowie im Rahmen der europäischen Strukturpolitik ab. Letztlich könnten über die Gründung eines Initiativkreises die Interessen der Regionen gebündelt sowie auf einen Anschluss an die europäische Diskussion ebenso wie auf eine aktive Einbindung in europäische Netzwerke hingewirkt werden.

Mit der grenzüberschreitenden Perspektive verbindet sich schließlich eine ,Europäisierung' von Politiken und Projekten, Akteuren und Institutionen auf allen Ebenen - notwendige Voraussetzung für ein Vorantreiben des Zieles der territorialen Kohäsion in Europa und einer bürgernahen Umsetzung der europäischen Idee.

Danksagung Die Autoren danken folgenden Personen für die Unterstützung im Rahmen der Bearbeitung: Dr. Horst Lutter und Dr. Rupert Kawka (Bundesinstitut für Bau-, Stadt- und Raumforschung (BBSR)), den MORO-Projektpartnern sowie Prof. Dr. Jürgen Aring (BfAG), Philippe Peters (Ministère du Développement durable et des Infrastructures, Luxemburg), Athéna Arendt (Metropolprojekt Saar-Moselle) und Sylvia Jost (Bundesamt für Raumentwicklung, Schweiz).

\section{Literatur}

AGEG (Arbeitsgemeinschaft Europäischer Grenzregionen) (2000): Praktisches Handbuch zur grenzübergreifenden Zusammenarbeit. Guide 2000 DT - Regionalpolitik. 3. Auflage. Online unter www. aebr.net/publikationen/pdfs/lace_guide.de.pdf (letzter Zugriff am 12.05.2009).

AGEG (Arbeitsgemeinschaft Europäischer Grenzregionen) (2004): Europäische Charta der Grenz- und grenzübergreifenden Regionen. Neufassung, Stand: 7. Oktober 2004. Gronau.

Ahlke, B.; Görmar, W.; Hartz, A. (2007): Territoriale Agenda der Europäischen Union und transnationale Zusammenarbeit. In: Informationen zur Raumentwicklung 7/8, 449-463.

BAK Basel Economics (2006): Das Oberrheingebiet als europäische Metropolregion. Studie im Auftrag der Oberrheinkonferenz. Basel.

Battis, U.; Kersten J. (2008): Europäische Politik des territorialen Zusammenhalts - Europäischer Rechtsrahmen und nationale Umsetzung. Rechtsgrundlagen im Rahmen der Allgemeinen Ressortforschung des Bundes. Herausgegeben vom Bundesamt für Bauwesen und Raumordnung und Bundesministerium für Verkehr, Bau und Stadtentwicklung. Bonn.
BBR (Bundesamt für Bauwesen und Raumordnung) (2005): Raumordnungsbericht 2005. Bonn. = Berichte des BBR, Bd. 21.

BBR (Bundesamt für Bauwesen und Raumordnung) (Hrsg.) (2008): Überregionale Partnerschaften. Ein MORO-Forschungsfeld. Bonn. = MORO-Informationen 3/1.

BBSR (Bundesinstitut für Bau-, Stadt- und Raumforschung) (Hrsg.) (2009): Überregionale Partnerschaften in grenzüberschreitenden Verflechtungsräumen. Ein MORO-Forschungsfeld. Bonn. = MORO-Informationen 5/1.

Beck, J. (2010): Grenzüberschreitende Zusammenarbeit als Gegenstand interdisziplinärer Forschung: Konturen eines wissenschaftlichen Arbeitsprogramms / La coopération transfrontalière, objet de recherche interdisciplinaire: Quelques réflexions sur un programme de travail scientifique. In: Wassenberg, B. (Hrsg.): Vivre et penser la coopération transfrontalière (Volume I): les régions frontalières françaises. Contributions du cycle de recherche sur la coopération transfrontalière de l'Université de Strasbourg et de l'Euro-Institut de Kehl. Stuttgart, 21-47.

BFS (Bundesamt für Statistik), Sektion Räumliche Analysen; Ernst Basler + Partner (2007): Überarbeitung der Agglomerationsdefinition. Grundlagenstudie zur Bestandesanalyse und Bedürfnisabklärung. Schlussbericht vom 29. Juni 2007. Zürich. Online unter http://www.bfs.admin.ch/bfs/portal/de/index/regionen/22/lexi. Document.105558.pdf (letzter Zugriff am 09.04.2009).

Blotevogel, H. H. (2007): Die Bedeutung der Metropolregionen in Europa. In: MIRaktuell Vierteljahresschrift 1, 7-10.

Blotevogel, H. H.; Schulze, K. (2009): Zum Problem der Quantifizierung der Metropolfunktionen deutscher Metropolregionen. In: Knieling, J. (Hrsg.): Metropolregionen. Innovation, Wettbewerb, Handlungsfähigkeit. Hannover, 30-58. = Forschungs- und Sitzungsberichte der ARL, Bd. 231.

BMVBS (Bundesministerium für Verkehr, Bau und Stadtentwicklung) (Hrsg.) (2010): Überregionale Partnerschaften in grenzüberschreitenden Verflechtungsräumen. Ein MORO-Forschungsfeld. Berlin. = MORO-Informationen 5/2.

BMVBS (Bundesministerium für Verkehr, Bau und Stadtentwicklung); BBR (Bundesamt für Bauwesen und Raumordnung) (2006): Perspektiven der Raumentwicklung in Deutschland. Bonn/Berlin. Online unter: www.bbr.bund.de/cln 005/nn 77088/BBSR/DE/ Veroeffentlichungen/Sonderveroeffentlichungen/2006/PerspektivenRaumentwicklg.html (letzter Zugriff am 09.04.2009).

DATAR (Délégation à l'aménagement du territoire et à l'action régionale) (2004): Appel à coopération métropolitaine. Pour un rayonnement européen des métropoles françaises. Paris. Online unter http://www.diact.gouv.fr/IMG/File/Appel_a_cooperation metropolitaine.pdf (letzter Zugriff am 20.04.2009).

DIACT (Délégation interministérielle à l'aménagement et à la compétitivité des territoires) (2005a): Actes des journées techniques de coopération métropolitaine. Paris. Online unter http://www.diact. gouv.fr/IMG/File/actes_journes_tec_2005.pdf (letzter Zugriff am 20.04.2009)

DIACT (Délégation interministérielle à l'aménagement et à la compétitivité des territoires (2005b): Résultats de l'appel à coopération métropolitaine. Paris. Online unter http://www.diact.gouv. fr/IMG/File/Carte090805ACmetropole\%20.pdf (letzter Zugriff am 20.04.2009).

Duhamelle, C.; Kossert, A.; Struck, B. (2007): Einleitung. Perspektiven für eine vergleichende Grenzforschung Europas. In: Duhamelle, C.; Kossert, A.; Struck, B. (Hrsg.): Grenzregionen. Ein europäischer Vergleich vom 18. bis zum 20. Jahrhundert. Frankfurt am Main, 9-21.

Europäische Kommission (1999): Europäisches Raumentwicklungskonzept EUREK. Auf dem Wege zu einer räumlich ausgewogenen und nachhaltigen Entwicklung der Europäischen Union. Angenommen beim Informellen Rat der für Raumordnung zuständigen Minister in Potsdam. Brüssel. 
European Commission (2001): Second report on economic and social cohesion. Unity, solidarity, diversity for Europe, its people and its territory. Adopted by the European Commission on 31 January 2001. Online unter http://ec.europa.eu/regional_policy/sources/ docoffic/official/reports/contentpdf en.htm (letzter Zugriff am 26.01.2009).

Le Gouvernement du Grand-Duché de Luxembourg (2004): IVL - Ein Integratives Verkehrs- und Landesentwicklungskonzept für Luxemburg. Die Idee, die Analyse, das Konzept. Ergebnisbroschüre. Luxemburg.

Hartz, A. (2009): Grenzüberschreitende Verflechtungsräume - Eine besondere Kategorie im Netz der europäischen Metropol- und Stadtregion. In: Planerin 1, 43-44.

Knippschild, R. (2009): Außer Spesen nichts gewesen? Nutzen und Erfolgsfaktoren grenzüberschreitender Kooperation in der Stadtund Regionalentwicklung im deutsch-polnisch-tschechischen Dreiländereck. In: Raumforschung und Raumordnung 67, 3, 228-238.

Köhler, S. (2007): Wachstumsregionen fernab der Metropolen Zusammenführung und Ausblick. In: Köhler, S. (Hrsg.): Wachstumsregionen fernab der Metropolen. Chancen, Potenziale und Strategien. Hannover, 116-118. = Arbeitsmaterial der ARL, Nr. 334.

Köhler, S. (2009): Großräumige grenzüberschreitende Verflechtungsräume in Deutschland - Weiterentwicklung der Leitbilder für die Raumentwicklung im aktuellen Programm „Modellvorhaben der Raumordnung“ (MORO) des Bundes. In: Standort - Zeitschrift für Angewandte Geographie 2, 33-39.

Kommission der Europäischen Gemeinschaft (2008): Grünbuch zum territorialen Zusammenhalt. Territoriale Vielfalt als Stärke. Mitteilung der Kommission an das Europäische Parlament, den Rat, den Ausschuss der Regionen und den europäischen Wirtschaftsund Sozialausschuss. Brüssel. Online unter http://ec.europa.eu/ regional_policy/consultation/terco/consultation_de.htm (letzter Zugriff am 26.01.2009).

Krocová, M. (2007): Kontinuität und Wandel. Die Wahrnehmung der sächsisch-böhmischen Grenze, 1780-1850. In: Duhamelle, C.; Kossert, A.; Struck, B. (Hrsg.): Grenzregionen. Ein europäischer Vergleich vom 18. bis zum 20. Jahrhundert. Frankfurt am Main, 181-202.

Levin, M. (2006): Der Eurodistrikt als Chance der grenzüberschreitenden Regionalplanung? Potenziale, Grenzen und Perspektiven der deutsch-französischen Regionalplanung am Beispiel des Eurodistrikt Region Freiburg / Centre et Sud Alsace. Unveröffentlichte Diplomarbeit an der Universität Hannover.
Ludwig, J.; Mandel, K.; Schwieger, C.; Terizakis, G. (2008): Abschließende Betrachtung. In: Ludwig, J.; Mandel, K.; Schwieger, C.; Terizakis, G. (Hrsg.): Metropolregionen in Deutschland. 11 Beispiele für Regional Governance. Baden-Baden, 184-188.

Manthey, G. (1992): Möglichkeiten der gemeinschaftlichen Regionalpolitik für die Entwicklung der Grenzregionen: Die INTERREGInitiative und andere begleitende Maßnahmen. In: Akademie für Raumforschung und Landesplanung (ARL) (Hrsg.): Grenzübergreifende Raumplanung - Erfahrungen und Perspektiven der Zusammenarbeit mit den Nachbarstaaten Deutschlands. Hannover, 31-44. = Forschungs- und Sitzungsberichte der ARL, Bd. 188.

Ministère de l'Intérieur, Direction de l'Aménagement du Territoire et de l'Urbanisme (2003): Grand Duché de Luxembourg. Programme Directeur d'Aménagement du Territoire. Luxembourg.

MOT (Mission Opérationnelle Transfrontalière) (2005): Espaces transfrontaliers. La lettre de la mission opérationnelle transfrontalière. $\mathrm{N}^{\circ} 20$, Octobre 2005. Paris. Online unter http://www.espacestransfrontaliers.org (letzter Zugriff am 20.05.2009).

ÖROK (Österreichische Raumordnungskonferenz) (Hrsg.) (2005): Europaregionen - Herausforderungen, Ziele, Kooperationsformen. Wien. ÖROK-Schriftenreihe Nr. 169.

Rumley, P.-A. (2008): Das Raumkonzept Schweiz - Wege für eine nachhaltige Raumentwicklung. In: Forum Raumentwicklung 3, 5-7.

Scherer, R.; Schnell, K.-D. (2002): Die Stärke schwacher Netzwerke. Entwicklung und aktuelle Situation der grenzüberschreitenden Zusammenarbeit in der Regio Bodensee. In: Europäisches Zentrum für Föderalismus-Forschung Tübingen (Hrsg.): Jahrbuch des Föderalismus 2002. Föderalismus, Subsidiarität und Regionen in Europa. Baden-Baden, 502-518.

Schmitt-Egner, P. (2005): Handbuch zur Europäischen Regionalismusforschung - theoretisch-methodische Grundlagen, empirische Erscheinungsformen und strategische Optionen des Transnationalen Regionalismus im 21. Jahrhundert. Wiesbaden.

Staeck, N. (1997): Politikprozesse in der Europäischen Union. Eine Policy-Netzwerkanalyse der europäischen Strukturfondpolitik. Baden-Baden. $=$ Nomos Universitätsschriften: Politik, Bd. 71 . 